\title{
Resección quirúrgica de metástasis pulmonares de cáncer colorrectal
}

\author{
Flores-Alvarez E*, Kelly-García J**, Ibarra-Pérez C** \\ Luna Pérez P*, Rodríguez-Ramírez $S^{*}$ \\ , .
}

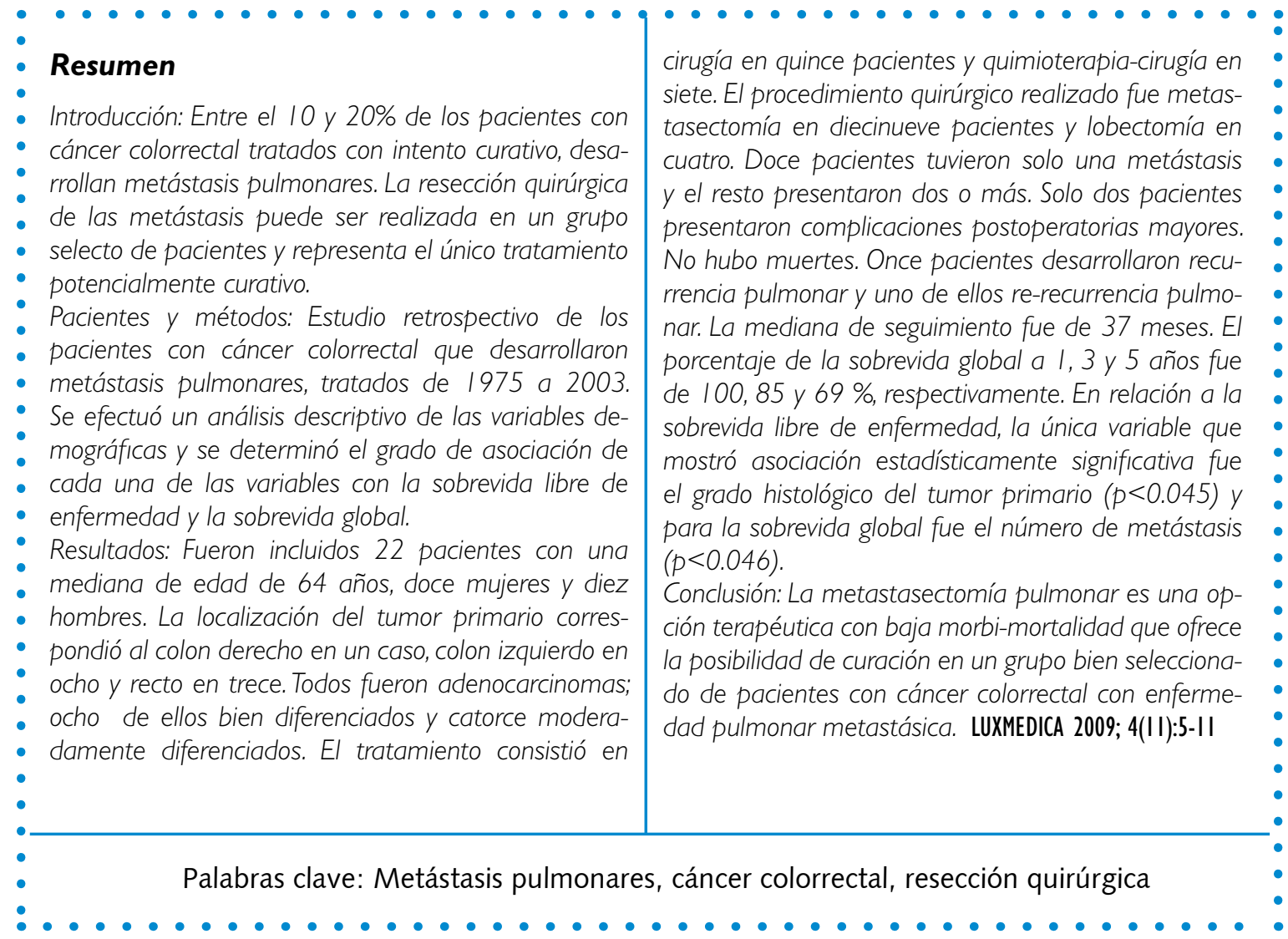

* Servicio de Tumores Colorrectales.

** Servicio de Tumores de Torax, División de Cirugía. Hospital de Oncología. Centro Médico Nacional Siglo XXI, México D.F. 


\section{Introducción}

El cáncer colorrectal constituye actualmente un problema de salud pública en el mundo. En los Estados Unidos es la cuarta neoplasia maligna más frecuente y la segunda causa de mortalidad por cáncer ${ }^{1}$. En México, en el cuatrienio 1993-1996 el IMSS notificó un total de 106,086 casos nuevos de neoplasias malignas, correspondiendo al colon, recto y ano 3,448 casos; en el Hospital de Oncología CMN Siglo XXI se concentró el 5.3\% $(5,669)$ del total de neoplasias; los tumores colorrectales representaron el $2.6 \%$, con 146 casos anuales ${ }^{2}$.

Se estima que un $70 \%$ de los cánceres colorrectales se presentan como enfermedad localizada y un $30 \%$ en forma avanzada. De los pacientes tratados con enfermedad localizada, un 50\% recae. El pulmón es el órgano extra-abdominal más frecuentemente involucrado en la diseminación hematógena ${ }^{3}$. De los pacientes sometidos a cirugía con intento curativo, un $10-20 \%$ desarrollan metástasis pulmonares en algún momento, de éstos solo un $10 \%$ presentan enfermedad metastásica limitada ${ }^{4,5}$. No existe evidencia de que el tratamiento sistémico con quimioterapia (QT) o la radioterapia (RT) mejore la sobrevida de estos pacientes por lo que la resección de las metástasis pulmonares es actualmente la única opción terapéutica útil ${ }^{4-8}$.

\section{| | | | | | | | | | | | | | | | | | | | | | | | | | | | | | | | | | | | | | | | | | | | | | | | | | | | | | | | | | | | | | | | | | | | | | | | | | | | | | | | | | | | | | | | | | | | | | | | | | | | | | | | | | | | | | | | | |}

\section{Material y métodos}

Se realizó una revisión retrospectiva del archivo del servicio de Cirugía de Tórax del Hospital de Oncología CMNS XXI para identificar a los pacientes con enfermedad metastásica pulmonar, tratados quirúrgicamente entre enero de 1975 y diciembre de 2003. Se seleccionaron los casos de cáncer colorrectal primario y se revisaron los expedientes clínicos. Las variables analizadas fueron la edad, el género, el tipo histológi$\mathrm{co}$, el nivel del antígeno carcinoembrionario $(A C E)$, el sitio y número de metástasis, el uso de tratamientos complementarios, el tipo de resección, la morbi-mortalidad operatoria, las recurrencias, la sobrevida libre de enfermedad y la sobrevida global. Para medir la fuerza de asociación de algunas variables con la sobrevida libre de enfermedad y la sobrevida global se utilizó la prueba de chi cuadrada, considerando una significancia estadística cuando el valor de p fue menor a 0.05. Se realizó un análisis de sobrevida global y sobrevida libre de enfermedad usando la prueba de KaplanMeier.

\section{| | | | | | | | | | | | | | | | | | | | | | | | | | | | | | | | | | | | | | | | | | | | | | | | | | | | | | | | | | | | | | | | | | | | | | | | | | | | | | | | | | | | | | | | | | | | | | | | | | | | | | | | | | | | | | | | | | | | |}

\section{Resultados}

Fueron incluidos 22 pacientes con un diagnóstico corroborado histológicamente de enfermedad pulmonar metastásica de primario colorrectal. La mediana de edad de los pacientes fue de 64 años (amplitud:
37-76 años); doce mujeres y diez varones. La localización del tumor primario correspondió a colon derecho en un caso, colon izquierdo en ocho y recto en trece. La histología fue de adenocarcinoma en todos los casos; ocho bien diferenciados y cator- 
ce moderadamente diferenciados. La etapa del primario de acuerdo a la clasificación de Astler-Coller fue: etapa $A$, uno; etapa B1, dos; etapa B2, siete; etapa B3, cuatro; etapa $C_{1}$, uno, y; etapa $C 2$, siete. Trece pacientes recibieron tratamiento adyuvante: dos con radioterapia (RT), nueve con quimioterapia (QT) y dos con RT y QT.

La enfermedad pulmonar metastásica fue diagnosticada en todos los pacientes por medio de una radiografía simple como estudio rutinario de seguimiento y corroborada por tomografía lineal en trece casos y por tomografía computarizada en ocho. Solo un paciente con metástasis pulmonares tuvo síntomas al momento del diagnóstico.

El tratamiento consistió en cirugía única en quince pacientes y quimioterapia-cirugía en siete. El procedimiento quirúrgico realizado fue toracotomía con metastasectomía en diecinueve pacientes y lobectomía en cuatro. Doce pacientes tuvieron solo una metástasis, cuatro tuvieron dos metástasis, cuatro presentaron tres lesiones, uno tuvo cuatro lesiones y uno cinco metástasis. Solo dos pacientes presentaron complicaciones postoperatorias mayores: insuficiencia cardiaca y fuga aérea. No hubo muertes durante el perioperatorio. La mediana de estancia hospitalaria fue de seis días (amplitud: 3-23 días). Once pacientes desarrollaron recurrencia pulmonar y uno de ellos re-recurrencia. El tratamiento de las recaídas pulmonares fue con cirugía única en ocho casos, QT en uno y QT-cirugía en tres. La cirugía consistió en metastasectomías en tres pacientes, lobectomía en cuatro, neumonectomía en dos y metastasectomías con lobectomía en uno.

El intervalo libre de enfermedad promedio entre el tratamiento del primario y el desarrollo de la primera enfermedad metastásica fue de 30 meses y entre el tratamiento de la metástasis y la recurrencia pulmonar fue de 24 meses. Siete pacientes desarrollaron metástasis extrapulmonares: dos en peritoneo, dos en hueso, dos en sistema nervioso central y uno en hígado.

La mediana de seguimiento fue de 37 meses; con una sobrevida libre de enfermedad en el 59.09\%. El porcentaje de la sobrevida global a 1, 3 y 5 años fue de $100,85.7$ y $69.23 \%$, respectivamente. Con relación a la sobrevida libre de enfermedad la variable que mostró asociación estadísticamente significativa fue el grado histológico del tumor primario $(p<0.045)$ y con respecto a la sobrevida global fue el número de metástasis $(p<0.046)$.

\section{Discusión}

El cáncer colorrectal es uno de los tumores malignos más frecuentes en el mundo, representa el $13 \%$ de las neoplasias malignas y es responsable del $10 \%$ de las muertes por cáncer en Estados Unidos. Al momento del diagnóstico dos terceras partes de los pacientes tienen enfermedad local o regional que puede ser tratada quirúrgicamente, de éstos el $50 \%$ recae. El hígado y el pulmón son los órganos más frecuentemente afectados por la diseminación hematógena. Un grupo pequeño de pacientes desarrolla metástasis aisladas, susceptibles de tratamiento quirúrgico ${ }^{9-11}$.

La presente serie incluye 22 pacientes tratados en la institución desde 1975, lo cual indica la baja frecuencia de su presentación aunado a los estrictos criterios de selección.

La mayoría de los enfermos con metástasis pulmonares candidatos a tratamiento quirúrgico son asintomáticos, solo uno de nuestros pacientes refirió síntomas. Generalmente se les diagnostica durante los controles posteriores a la cirugía, o a la administración de quimio o radioterapia. Cuando los enfermos están sintomáticos generalmente tienen lesiones irresecables ${ }^{12}$.

La extensión de la resección quirúrgica ha cambiado considerablemente en los últimos años con una tendencia a realizar procedimientos resectivos menores para 
preservar la mayor cantidad posible de tejido pulmonar funcional. Un margen de uno a dos centímetros es considerado suficiente para la mayoría de las lesiones. Las lesiones periféricas se pueden tratar casi siempre con una resección en cuña con pinzas y sutura manual o con engrapadoras, o por medio de metastasectomía precisa con electrocauterio; las lesiones centrales pueden requerir de lobectomía o, en algunos casos, hasta neumonectomía ${ }^{13}$.

La sobrevida a 5 años en los diferentes reportes publicados en la literatura mundial fluctúa entre 10 y $60 \%{ }^{14,15}$. La sobrevida en nuestro grupo fue de $77 \%$ con una mediana de seguimiento de 37 meses; la sobrevida actuarial esperada a 5 años debe ser comparable a la observada en la mayoría de centros oncológicos y cardiotorácicos.

La influencia de diversos factores en la sobrevida ha sido objeto de gran controversia, algunos autores reportan que el intervalo libre de enfermedad entre el tumor primario y la recurrencia, el sitio y la etapa del cáncer colorrectal primario, el patrón histológico del tumor primario, los niveles preoperatorios de $A C E$, el número, la extensión y localización de la lesión metastásica y la extensión de la resección de la metástasis son factores que pueden influir de manera significativa en la sobrevida. Temple y Ketcham ${ }^{16}$ reportaron una reducción entre 10 y $40 \%$ en la sobrevida a 5 años de pacientes con un periodo libre de enfermedad menor de un año en comparación con un periodo libre de enfermedad mayor a 5 años. Girard ${ }^{17}$ reportó una sobrevida de $16 \%$ en pacientes con niveles preoperatorios de ACE elevados, mientras que la sobrevida fue de $47 \%$ en pacientes con niveles de ACE preoperatorios normales. Zanella ${ }^{18}$ observó que una resección radical fue el único factor pronóstico independiente para la sobrevida libre de enfermedad, aunque no tuvo relevancia en la sobrevida global. Al analizar las variables más relevantes en nuestro estudio, como son la edad, el grado histológico, la localización y la etapa del tumor primario, los niveles de ACE preoperatorio, el intervalo libre de enfermedad, el número de metástasis, el empleo de quimioterapia adyuvante y la recurrencia pulmonar, observamos que la única variable que mostró asociación estadísticamente significativa con la sobrevida libre de enfermedad, fue el grado histológico del tumor primario y con respecto a la sobrevida global la única variable que alcanzó significancia estadísti$\mathrm{ca}$, fue el número de metástasis.

El pulmón fue el sitio más frecuente de recurrencia después de una resección pulmonar de metástasis. Es recomendable un seguimiento cuidadoso de los pacientes para detectar enfermedad recurrente en una fase temprana. McAffe ${ }^{19}$ recomienda tomar una telerradiografía de tórax, una tomografía computarizada del tórax y la medición del ACE en forma semestral; con un seguimiento de por vida debido a que las metástasis pulmonares recurrentes pueden aparecer después de muchos años. La detección oportuna de la recurrencia pulmonar permitió realizar una segunda resección pulmonar en forma exitosa y que no tuvo influencia negativa en la sobrevida global.

\section{Cuadro I}

\section{Niveles promedio de ACE en 22 pacientes con metástasis pulmonares por cáncer colorrectal}

\begin{tabular}{|lcc|}
\hline & Preoperatorio $\mathbf{( n g / m l )}$ & Postoperatorio (ng/ml) \\
\hline Tumor colorrectal & 15.60 & 2.76 \\
\hline Metástasis pulmonar & 6.23 & 3.41 \\
\hline Recurrencia pulmonar & 5.78 & 2.24 \\
\hline & & \\
\hline
\end{tabular}




\section{Cuadro 2}

\section{Asociación de las variables con la sobrevida libre de enfermedad}

\begin{tabular}{|c|c|c|}
\hline \multirow[t]{2}{*}{ Variable } & \multicolumn{2}{|c|}{ Sobrevida libre de enfermedad } \\
\hline & $\%$ & $\mathrm{p}$ \\
\hline \multicolumn{3}{|l|}{ Edad } \\
\hline$<60$ años & 70 & \\
\hline$>60$ años & 50 & 0.353 \\
\hline \multicolumn{3}{|l|}{ Localización del primario } \\
\hline Colon derecho & 100 & \\
\hline Colon izquierdo & 25 & \\
\hline Recto & 76 & 0.082 \\
\hline \multicolumn{3}{|l|}{ Etapa clínica del primario } \\
\hline$A$ & 100 & \\
\hline $\mathrm{B}$ & 46 & \\
\hline $\mathrm{C}$ & 75 & 0.315 \\
\hline \multicolumn{3}{|c|}{ Grado histológico del primario } \\
\hline Bien diferenciado & 100 & \\
\hline Mod. Diferenciado & 43 & 0.045 \\
\hline \multicolumn{3}{|l|}{ ACE preoperatorio } \\
\hline \multicolumn{3}{|l|}{ Normal } \\
\hline Aumentado & 50 & 0.095 \\
\hline \multicolumn{3}{|c|}{ Periodo libre de enfermedad } \\
\hline$<36$ meses & 60 & \\
\hline$>36$ meses & 57 & 0.901 \\
\hline \multicolumn{3}{|l|}{ QT adyuvante } \\
\hline $\mathrm{Si}$ & 71 & \\
\hline No & 61 & 0.432 \\
\hline \multicolumn{3}{|l|}{ Extensión de la resección } \\
\hline Metastasectomía & 67 & \\
\hline Otro & 25 & 0.134 \\
\hline \multicolumn{3}{|l|}{ Número de metástasis } \\
\hline 1 & 75 & \\
\hline$>1$ & 40 & 0.104 \\
\hline \multicolumn{3}{|l|}{ Recurencia pulmonar } \\
\hline $\mathrm{Si}$ & 63 & \\
\hline No & 54 & 0.672 \\
\hline
\end{tabular}




\section{Cuadro 3}

\section{Asociación de las variables con la sobrevida global}

\begin{tabular}{|c|c|c|}
\hline \multirow[t]{2}{*}{ Variable } & \multicolumn{2}{|c|}{ Sobrevida global } \\
\hline & $\%$ & $\mathrm{p}$ \\
\hline \multicolumn{3}{|l|}{ Edad } \\
\hline$<60$ años & 80 & \\
\hline$>60$ años & 75 & 0.658 \\
\hline \multicolumn{3}{|l|}{ Localización del primario } \\
\hline Colon derecho & 100 & \\
\hline Colon izquierdo & 63 & \\
\hline Recto & 85 & 0.740 \\
\hline \multicolumn{3}{|l|}{ Etapa clínica del primario } \\
\hline$A$ & 100 & \\
\hline $\mathrm{B}$ & 76 & \\
\hline $\mathrm{C}$ & 75 & 0.552 \\
\hline \multicolumn{3}{|c|}{ Grado histológico del primario } \\
\hline Bien diferenciado & 100 & \\
\hline Mod. Diferenciado & 64 & 0.169 \\
\hline \multicolumn{3}{|l|}{ ACE preoperatorio } \\
\hline \multicolumn{3}{|l|}{ Normal } \\
\hline Aumentado & 60 & 0.129 \\
\hline \multicolumn{3}{|c|}{ Periodo libre de enfermedad } \\
\hline$<36$ meses & 73 & \\
\hline$>36$ meses & 71 & 0.953 \\
\hline \multicolumn{3}{|l|}{ QT adyuvante } \\
\hline $\mathrm{Si}$ & 71 & \\
\hline No & 84 & 0.214 \\
\hline \multicolumn{3}{|l|}{ Extensión de la resección } \\
\hline Metastasectomía & 89 & \\
\hline Otro & 25 & 0.474 \\
\hline \multicolumn{3}{|l|}{ Número de metástasis } \\
\hline 1 & 83 & \\
\hline$>1$ & 70 & 0.046 \\
\hline \multicolumn{3}{|l|}{ Recurencia pulmonar } \\
\hline $\mathrm{Si}$ & 90 & \\
\hline No & 63 & 0.544 \\
\hline
\end{tabular}




\section{Gráfica I}

\section{Sobrevida acumulada por la prueba de Kaplan-Meier}

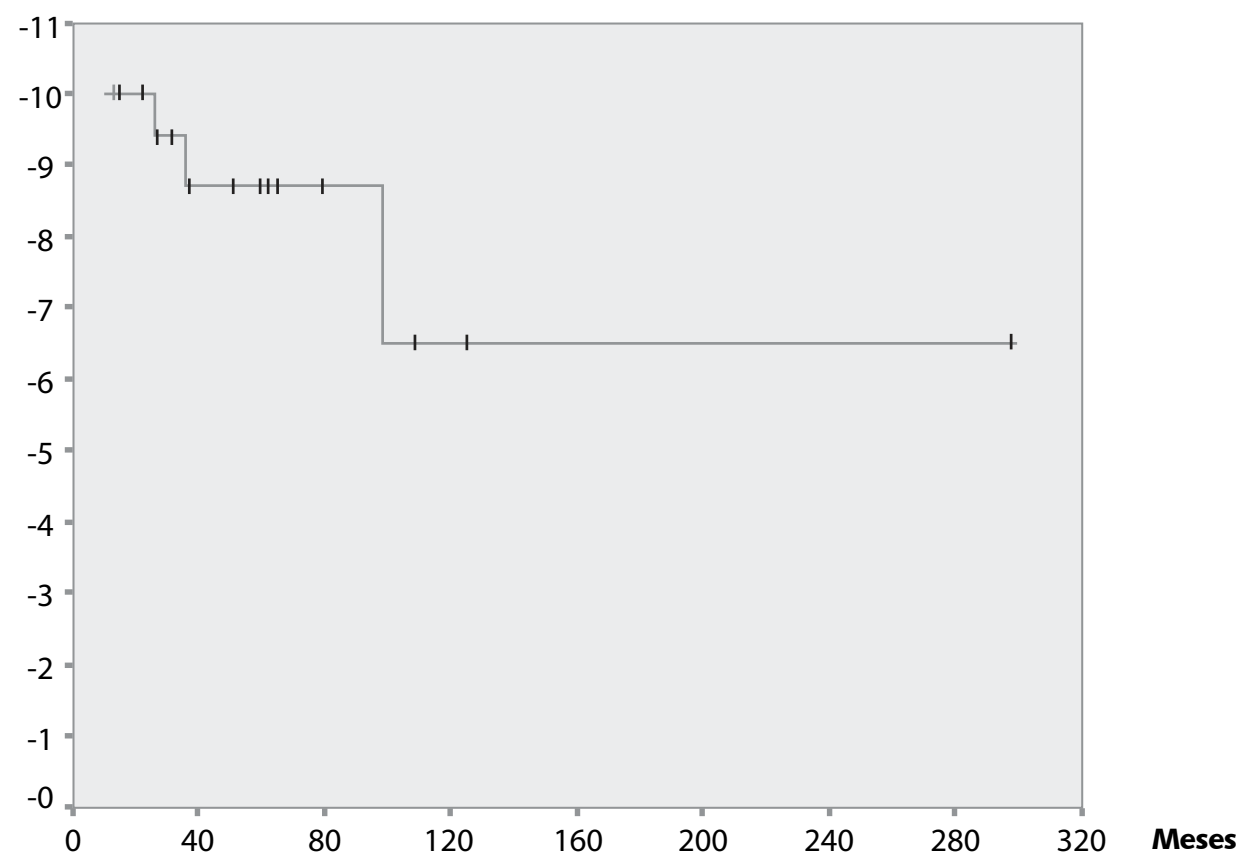

\section{Bibliografía}

1 Jemal A, Siegel R, Ward E et al. Cancer Statistics, 2006. CA Cancer J Clin 2006;56:106-130.

2 Rodríguez-Cuevas S, Labastida Almendro S, Tapia Conyer R, Kuri Morales P, Macias Martinez CG. Registro Histopatológico de Neoplasias en México, Población derechohabiente del IMSS 1993-1996. 1era ed. 1999

3 van Schaik PM, Kouwenhoven EA, Bolhuis RJ et al. Pulmonary resection for metastases from colorectal cancer. J Thorac Oncol 2007;2(7):652-656.

4 Maricola FM, Mark JBD. Selection factors resulting in improved survival after surgical resection of tumors metastatic to the lung. Arch Surg 1990;125:13871393.

5 Inoue $M$, Ohta $M$, luchi $K$ et al. Benefits of surgery for patients with pulmonary metastases for colorectal carcinoma. Ann Thorac Surg 2004;78(1):238-44.

6 Granados GM, Green SL. Tratamiento de la enfermedad metastásica pulmonar por cáncer colorrectal. Cir Gen 2000;22:172-176.

7 Shiono S, Ishii G, Nagai K et al. Histopathologic prognostic factors in resected colorectal lung metastases. Ann Thorac Surg 2005;79(1):278-282.

8 Saito $\mathrm{Y}$, Omiya $\mathrm{H}$, Kohno $\mathrm{K}$ et al. Pulmonary metastasectomy for 165 patients with colorectal carcinoma: A prognostic assessment. J Thorac Cardiovasc Surg. 2002;124(5):1007-13.

9 Zink S, Kayser G, Gabius HJ et al. Survival, diseasefree interval, and associated tumor features in patients with colon/rectal carcinomas and their resected intra-pulmonary metastases. Eur J Cardiothorac Surg 2001;19(6):908-913
10 Mountain CF, McMurtrey MJ, Hermes KE. Surgery for pulmonary metastasis: A 20-year experience. Ann Thorac Surg 1984;38:323-329.

11 Kolodziejski L, Góralczyk J, Dyczek S, Duda K, Nabialek $\mathrm{T}$. The role of surgery in lung metastases. Eur J Surg Oncol 1999; 25:410-417.

12 Rena O, Casadio C, Viano $\mathrm{F}$ et al. Pulmonary resection for metastases from colorectal cancer: factors influencing prognosis. Twenty-year experience. Eur J Cardiothorac Surg 2002;21(5):906-12

13 Sakamoto T, Tsubota N, Iwanaga K et al. Pulmonary resection for metastases from colorectal cancer. Chest 2001;119(4):1069-72.

14 lizasa $T$, Suzuki $M$, Yoshida $S$ et al. Prediction of prognosis and surgical indications for pulmonary metastasectomy from colorectal cancer. Ann Thorac Surg 2006;82(1):254-60

15 Shiono S, Ishii G, Nagai K et al. Histopathologic prognostic factors in resected colorectal lung metastases. Ann Thorac Surg 2005;79(1):278-82.

16 Temple WK, Ketcham AS. Surgical management of pulmonary metastases. Sem Oncol 1980;7:468480.

17 Girard P, Baldeyrou P, Le Chevalier T, Le Cesne A, Brigandi A, Grunenwald D. Surgery for pulmonary metastases. Cancer 1994;74:2791-2797.

18 Zanella A, Marchet A, Mainente P et al. Resection of pulmonary metastases from colorectal carcinoma. Eur J Surg Oncol. 1997;23(5):424-7.

19 McAfee MK, Allen MS, Trastek VF, Ilstrup DM, Deschamps C, Pairolero PC. Colorectal lung metastases: Result of surgical excision. Ann Thorac Surg 1992;53:780-786. 